\title{
The local structure in liquid methylamine and methylamine-water mixtures
}

\author{
Peter G. Kusalik \\ Department of Chemistry, Dalhousie University, Halifax, Nova Scotia, B3H 4J3, Canada \\ Dan Bergman and Aatto Laaksonen ${ }^{\text {a) }}$ \\ Division of Physical Chemistry, Arrhenius Laboratory, Stockholm University, SE-106 91 Stockholm, Sweden
}

(Received 14 February 2000; accepted 14 August 2000)

\begin{abstract}
Molecular dynamics (MD) computer simulations are carried out on liquid methylamine and on 10 and $30 \mathrm{wt} \%$ aqueous solutions of methylamine. The local three-dimensional structure in these liquid systems is investigated using standard one-dimensional radial distribution functions and the fully three-dimensional measure of the local structure around the molecule, spatial distribution functions. Time correlation functions for the linear and angular motion of methylamine in the molecular coordinate system are also explored. From this analysis, a detailed structural picture emerges, revealing local molecular environments in these liquids that are both complex and varied. Hydrogen bond balance is found to play a key role in determining preferred arrangements. Strong hydrogen bonds are formed around the amino group in the pure liquid and in aqueous solution. At the same time, there is a strong hydrophobic association of methyl groups. The hydration structure in aqueous solution is found to be particularly rich, where in addition to the usual H-bonding nearest neighbors in the first hydration shell of the amino group, there are bridging water molecules and a novel type of distinctly non-H-bonding neighbors. The hydration structure in aqueous methylamine solution differs substantially from that found in methanol-water liquid mixtures. () 2000 American Institute of Physics. [S0021-9606(00)51142-8]
\end{abstract}

\section{INTRODUCTION}

The study of small functionalized organic molecules in aqueous solution is a useful first step towards gaining a basic understanding of the behavior of biomolecular systems in their natural aqueous environments. Computer simulations represent an extremely useful tool in these investigations. Increased knowledge of long-range (ionic and polar) interactions, intermolecular hydrogen bonding to water (solvent) molecules, and the entire three-dimensional hydration structure, including both hydrophilic and hydrophobic regions, are all applicable when the role of water in biological processes is investigated. ${ }^{1-3}$ Also, there are several distinct advantages in first studying aqueous solutions of small molecules before starting to model complex macromolecules of thousands of atoms. For example, the simulation results are statistically reliable because several solute molecules can be included in the solution and the simulations can be extended to cover relatively long periods of time with relatively modest computer resources. Also, there are often accurate experimental data available, which can be used to refine the molecular models and force fields. The analysis of simulation data is more straightforward with fewer degrees of freedom to include. Hence, a thorough and systematic investigation of a set of selected small molecules provides a good opportunity to conceptually build large biomolecules from small molecular units rather than plain atoms.

This approach has been used in our laboratory during the last ten years. We have carried out studies of aqueous solu-

\footnotetext{
a) Author to whom correspondence should be addressed. Electronic mail: aatto@tom.fos.su.se
}

tions of benzene, ${ }^{4}$ methanol, ${ }^{5}$ nitriles, ${ }^{6-9}$ methane, ${ }^{10}$ nitrates, ${ }^{11}$ and electrolytes. ${ }^{12}$ Our aim has been to study the solvation structure and dynamics as well as the molecular motion in solution and the solute-solvent interactions, and to compare these results with NMR measurements made on the same systems. In many cases the effects of concentration were examined. In these simulations the small molecules were kept rigid, a reasonable simplification when investigating solvation phenomena, and the simulation results have been compared with experimental data ${ }^{13}$ using motional models. For large molecules, of course, flexibility is usually included, but typically where the molecule has been divided into small rigid units. When these units are detached and terminated they can correspond to small molecules (e.g., methanol, methylamine, etc.). In our attempts to examine biomolecules in this way, we have studied carboxylic acids, ${ }^{14}$ carbohydrates,${ }^{15}$ nucleotides, ${ }^{16}$ and DNA,${ }^{17}$ to give a few examples.

In this work we have considered the hydration of the amino functional group by investigating (mono)methylamine $\left(\mathrm{CH}_{3} \mathrm{NH}_{2}\right)$, dissolved in liquid water. $\mathrm{CH}_{3} \mathrm{NH}_{2}$ is a gas at room temperature $\left(T_{b}=-6^{\circ} \mathrm{C}\right)$. Commercially, methylamine is sold as a $30 \%$ by weight aqueous solution, because the vapor is extremely flammable and hazardous. Also the binary azeotrope of water and methylamine has roughly this composition and has a freezing point minimum of $-43{ }^{\circ} \mathrm{C} .{ }^{18}$

Amines play important roles in chemistry and biology. Together with the common $\mathrm{O}-\mathrm{H} \cdots \mathrm{O}$ hydrogen bonds, the $\mathrm{N}-\mathrm{H} \cdots \mathrm{O}$ (and certainly $\mathrm{N}-\mathrm{H}^{+} \ldots \mathrm{O}$ ) hydrogen bonds are important factors influencing conformational structures and crystals of biomolecules. The hydration of amines is known 
to influence their basicity in aqueous solution. ${ }^{19,20}$ Still, very few computer simulation studies of aqueous amine solutions have been reported. Reports on similar systems include the Monte Carlo studies of dilute solutions of methylamine in water by Dunn et al. ${ }^{21,22}$ and Nagy et $a l .{ }^{23}$ A hybrid RISMSCF study of methylamines in aqueous solution investigated behavior connected to their basicity anomaly. ${ }^{24}$ Several simulation studies ${ }^{25-30}$ have focused upon the solvation free energies of simple amines and their irregular ordering [i.e. $\left.\mathrm{CH}_{3} \mathrm{NH}_{2}<\mathrm{NH}_{3} \approx\left(\mathrm{CH}_{3}\right)_{2} \mathrm{NH}<\left(\mathrm{CH}_{3}\right)_{3} \mathrm{~N}\right]$.

This work is a part of a series of investigations where we focus on the spatial solvation structure within selected, strongly associated, hydrogen-bonded liquids and solutions. Our primary tools in this work are three-dimensional atomic densities, known as the spatial distribution functions. ${ }^{31,3}$ The liquids and solutions studied so far using this approach include water, ${ }^{32}$ methanol, ${ }^{33}$ water-methanol mixtures, ${ }^{5}$ water-acetonitrile mixtures, ${ }^{7-9}$ and a water-DMSO mixture. ${ }^{15}$ We have shown that the spatial distribution functions (SDF) provide a very detailed picture of the solvation structure. For example, they make it possible to define much more clearly and quantitative a "hydrogen-bond" and allow the investigation of the average local environment around a molecule within a liquid. ${ }^{7,9,34}$ Because of their richness in detail and the fact that these are four-dimensional functions (three space coordinates plus the probability intensity), their visualization requires some special attention. Various graphical techniques to illustrate and to extract both qualitative and quantitative information from SDFs have been discussed previously. 8,3

In Sec. II the molecular models and computational details are given in more detail. Our results are presented and discussed in Sec. III, while our conclusions are given in Sec. IV.

\section{MOLECULAR MODELS AND COMPUTATIONAL DETAILS}

Methylamine is readily soluble in water. In this work we have studied two concentrations, corresponding to approximately 10 and $30 \mathrm{wt} \%$ solutions of methylamine at $20{ }^{\circ} \mathrm{C}$. The $10 \mathrm{wt} \%$ mixture is relatively dilute and so still fairly ideal, while several excess properties have their extreme points close to $30 \mathrm{wt} \%$. The freezing point, for example, has its minimum of $-43{ }^{\circ} \mathrm{C}$ at 28 wt $\%$ of methylamine. ${ }^{18}$ The total of 256 molecules were used in each simulation, of which 14 and 38, respectively, were solute molecules for the two solutions. For the molecular interaction potentials we have used the three-site simple point charge model, SPC/E, of Berendsen and co-workers ${ }^{35}$ for water, and the four-site model of Impey et al. ${ }^{36}$ for methylamine. In the methylamine model the methyl group is represented by a united- $\mathrm{CH}_{3}$ particle, a common simplification used to reduce the number of interaction sites. The inert methyl group is then reasonably described as a Lennard-Jones sphere. This approximation is used in several other potential models, including the threesite model for methanol of Jorgensen ${ }^{37}$ and the three-site model for acetonitrile of Jorgensen and Briggs. ${ }^{38}$ Both of these models give good structural, thermodynamical, and dynamical results for the pure liquids; since our discussion of
TABLE I. Potential models and molecular geometries.

\begin{tabular}{lllc}
\hline \hline & $\epsilon(\mathrm{kcal} / \mathrm{mol})$ & $\sigma(\AA)$ & $q($ in units of $e)$ \\
\hline $\mathrm{H}_{2} \mathrm{O}^{\mathrm{a}}$ & & & \\
$\mathrm{O}$ & 0.1554 & 3.1656 & -0.8476 \\
$\mathrm{H}$ & 0.0 & 0.0 & +0.4238 \\
& & & \\
$\mathrm{CH}_{3} \mathrm{NH}_{2}{ }^{\mathrm{b}}$ & & & 0.25 \\
$\mathrm{Me}$ & 0.170 & 3.80 & -1.05 \\
$\mathrm{~N}$ & 0.170 & 3.30 & 0.40 \\
$\mathrm{H}$ & 0.00 & 0.00 & \\
\hline \hline
\end{tabular}

${ }^{\mathrm{a}} \mathrm{R}(\mathrm{O}-\mathrm{H}): 1.00 \AA, \angle(\mathrm{H}-\mathrm{O}-\mathrm{H}): 109.47^{\circ}$

${ }^{\mathrm{b}} \mathrm{R}(\mathrm{Me}-\mathrm{N}): 1.456 \AA, \mathrm{R}(\mathrm{N}-\mathrm{H}): 1.033 \AA, \angle(\mathrm{H}-\mathrm{N}-\mathrm{H}): 104.4^{\circ}, \angle(\mathrm{Me}-\mathrm{N}-$ $\mathrm{H}): 121.6^{\circ}$.

the structure will focus on qualitative features which should be sensitive to the basic natures of the intermolecular potentials but not their details, our results will be relatively invariant to this choice. Furthermore, because of the united atom description, these molecules can be treated as rigid without taking into account the methyl rotation, which we assume plays a minor role in determining the hydration structure. Methylamine has an additional internal degree of freedom, inversion, but this motion is also not considered in the present work. We remark that this model for methylamine was used successfully by Impey et $a l .{ }^{36}$ to study the solvation of $\mathrm{Li}^{+}$and $\mathrm{Cl}^{-}$ions.

The potential parameters and the molecular geometries are given in Table I, and the molecular coordinate systems used in the present work for methylamine is defined in Fig. 1. We obtain a molecular coordinate system from the diagonalization of the moment of inertia tensor, where the principal axis of methylamine lies approximately along the $z$-axis. In our definition of the local frame for water, the molecule lies in the $X Z$-plane, with the dipole axis coinciding with the $Z$-axis. These local coordinate systems will be important in the analysis of our results in Sec. III.

Periodic boundary conditions were employed in our simulations and the solute-solvent cross-interactions are calculated using the Lorentz-Berthelot combination rules. ${ }^{39}$ The Coulombic interactions were treated by the Ewald method $^{39}$ and the short-range interactions were truncated at
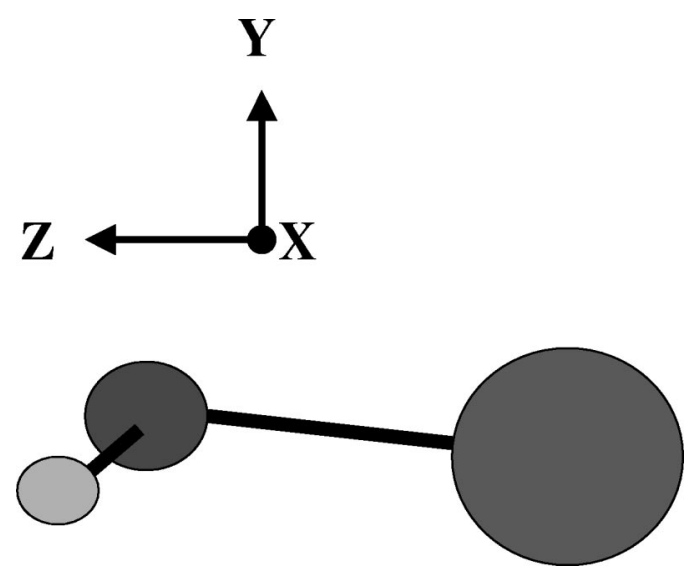

FIG. 1. Local molecular coordinate system (principal frame) for methylamine used in this work. 
TABLE II. Characteristic simulation parameters.

\begin{tabular}{lccc}
\hline \hline \multicolumn{1}{c}{ Quantity } & $\begin{array}{c}10 \mathrm{wt} \% \text { of } \\
\mathrm{MeNH}_{2}\end{array}$ & $\begin{array}{c}30 \mathrm{wt} \% \text { of } \\
\mathrm{MeNH}_{2}\end{array}$ & $\begin{array}{c}\text { Pure liquid } \\
\mathrm{MeNH}_{2}\end{array}$ \\
\hline $\mathrm{Nr} . \mathrm{MeNH}_{2}$ & 14 & 38 & 256 \\
$\mathrm{Nr} . \mathrm{H}_{2} \mathrm{O}$ & 242 & 218 & $\ldots$ \\
Temp. $(\mathrm{K})$ & 293 & 293 & 250 \\
$\rho\left(\mathrm{g} / \mathrm{cm}^{3}\right)$ & 0.974 & 0.926 & 0.66 \\
Cutoff $(\AA)$ & 10.07 & 10.46 & 13.558 \\
Simulation (ns) & 0.5 & 0.5 & 0.2 \\
\hline \hline
\end{tabular}

$10 \AA$ A. The simulation software used was a modified version of McMoldyn. ${ }^{40}$ The density and temperature and some other relevant simulation parameters are given in Table II. We remark that experimental densities were used in our simulations.

The simulations were carried out on a Fujitsu VX vector parallel supercomputer at the Center for Parallel Computers at the Royal Institute of Technology (KTH) in Stockholm, and on the departmental Linux pc cluster at the division of Physical Chemistry, Stockholm University.

\section{RESULTS AND DISCUSSION}

The purpose of the present work is to investigate the solvation structure in aqueous solution for a simple molecule that is a double donor and single acceptor of hydrogen bonds. Further motivation for this work is provided by the fact that amino groups occur frequently, for example, in amino acids and many molecules with pharmaceutical potential, as well as in many hazardous compounds. By studying a small molecule such as methylamine, we hope to be able to provide insights into the hydration structure of larger molecules containing amino groups. Moreover, a detailed understanding of the hydration and thermodynamics of hydration (e.g., free energies) of molecules such as methylamine will only be possible once we have resolved the underlying structure and structural changes that determine them.

Radial distribution functions (RDF) have been the traditional way to study structure in pure liquids and solutions because they can be obtained directly from the Fourier transforms of structure factors available from diffraction experiments. ${ }^{39,41}$ RDFs are functions of interparticle separations only, and hence ignore the orientation of the local frame. This is a rather crude simplification, as will be discussed in the next section, however they are still widely used because they can be easily constructed in a computer simulation from the pairwise interatomic distances. When the RDFs for several molecular sites are combined together, the RDFs can often provide some insights into the immediate environments of those atoms interacting strongly with other molecules, for example as in hydrogen $(\mathrm{H})$ bonding events. In this article we will include analysis based on the RDFs in order to make comparisons with previous work and to try to extract a general picture of the hydration. However, we will show that spatial distribution functions (SDF) provide a much more detailed and considerably less ambiguous picture of the local structural environment in solution (albeit at the pair level), especially if one wishes to understand the pos-

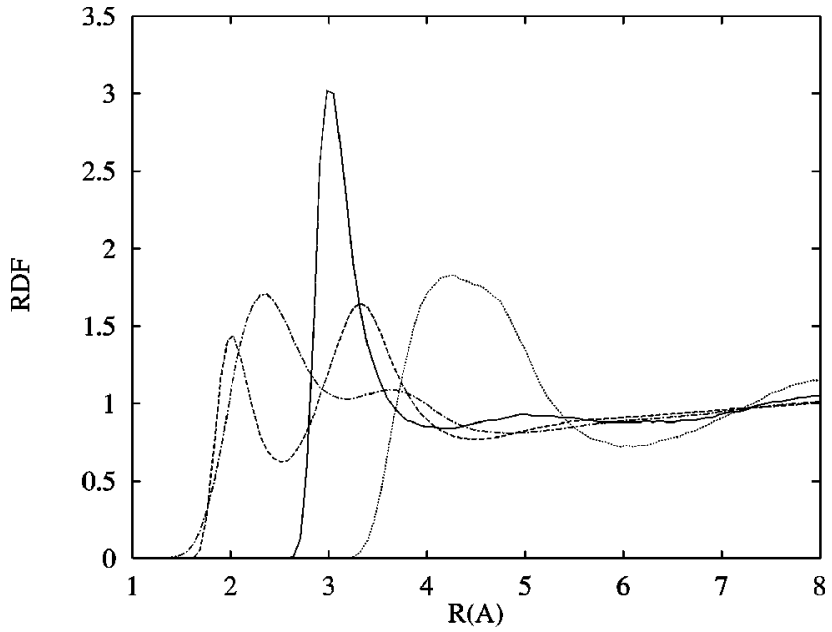

FIG. 2. Radial distribution functions for pure liquid methylamine. The solid, dashed, dotted, and dashed-dotted lines represent the nitrogen-nitrogen, nitrogen-hydrogen, methyl-methyl, and hydrogen-hydrogen site-site RDFs, respectively.

sible arrangements of the solutes and the solvent (water) and how the solutes interact with each other in solution.

It is important to point out that as a consequence of their three-dimensional nature, it is not possible to view fully the information contained in a single SDF; rather only slices through this data set can be visualized. In this article we will show isosurface (constant probability density) representations, where the thresholds have been carefully selected to best represent the principal structural features. A SDF analysis can be made even more powerfully by overlapping the density features of two (or more) types of neighboring sites. This can, for example, provide an immediate indication of the orientation of a neighboring molecule. This approach has been used extensively in our analysis (as viewed on a color graphics computer console), however for clarity most of the SDF plots included in this article will display only a single function.

\section{A. Structure in pure liquid methylamine}

In Fig. 2, the radial distribution functions between several site pairs are shown for pure liquid methylamine. The nitrogen-nitrogen $(\mathrm{N}-\mathrm{N})$ RDF shows a relatively sharp first peak at $3 \AA$ which then drops into an unusually broad minimum with only a small shoulder at $5 \AA$. The N-H RDF has two clear peaks, one at $2 \AA$, due presumably to $\mathrm{H}$-bonding, and a second at $3.3 \AA$. We see in Fig. 2 that the $g\left(r_{\mathrm{HH}}\right)$ has a rather broad first peak at $2.5 \AA$ with a strong shoulder at 3.7 $\AA$. Our results for the RDFs are in good agreement with those of Impey et al. ${ }^{36}$ at $-10^{\circ} \mathrm{C}$, who also remarked that these functions are very reminiscent of the corresponding RDFs for liquid methanol. However, we will show below that the SDFs reveal that the local structure within the two liquids differ considerably.

Analysis of the coordination numbers begins to suggest that there may be some unexpected features in the local structure. Integration of the first peak in $g\left(r_{\mathrm{NH}}\right)$ gives a coordination number of essentially unity, indicating that, like in liquid methanol, a methylamine makes on average only two 

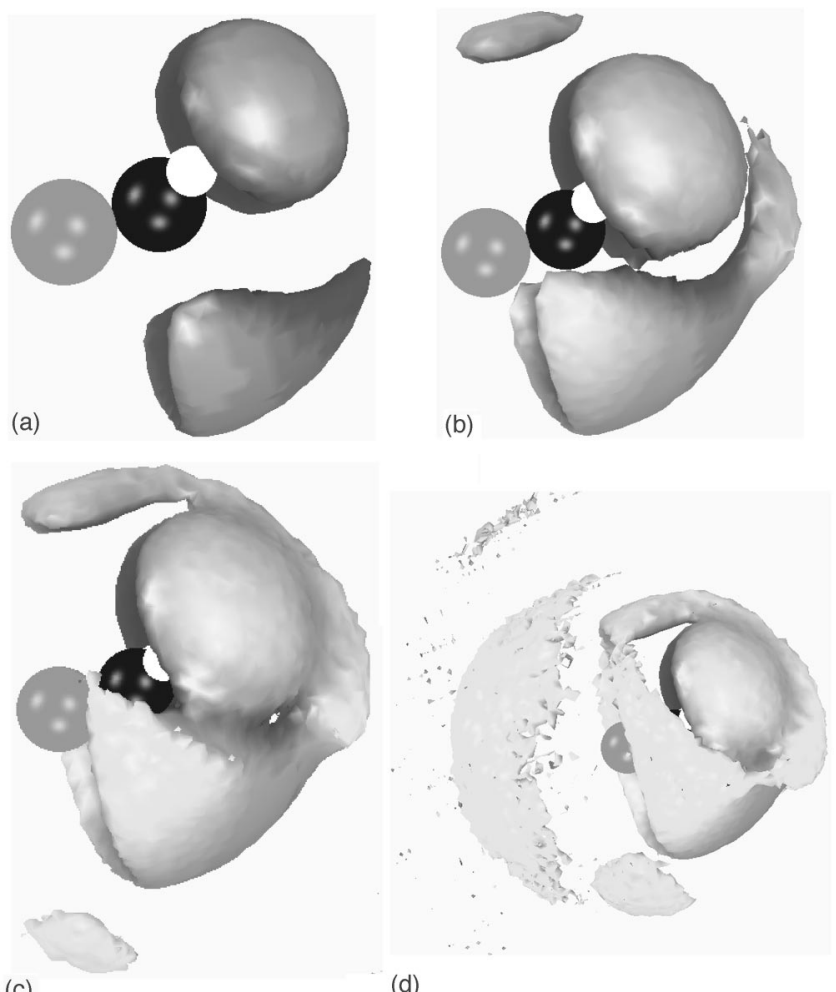

(c)

(d)

FIG. 3. Local structure around a methylamine molecule in pure liquid methylamine as measured by the nitrogen-nitrogen SDF. The isosurfaces in (a) represent a nitrogen density 2.5 times that of the bulk, in (b) 1.5 , in (c) 1.3 , and in (d) 1.18 times the bulk value. Note that in each case the orientation of the central molecule is unchanged. The surfaces have been shaded so that those appearing lighter are further from the nitrogen.

(strong) H-bonds. Thus, although methylamine possesses three H-bonding sites, the constraint of H-bond balance dictates that one of the donor sites remain unutilized. Integrating over the second peak in $g\left(r_{\mathrm{NH}}\right)$ finds an additional eight neighboring hydrogen atoms. Yet, the coordination number from the first peak in the N-N RDF is about 4, although this value is somewhat sensitive to the choice of the point of termination of the integration. Clearly, not all the near neighbors in the first peak in $g\left(r_{\mathrm{NN}}\right)$ are H-bonded, but their identity is otherwise not resolvable from the RDFs.

In Fig. 3, we explore the local structure around a methylamine molecule through the N-N SDF. The isosurfaces associated with four different thresholds, 2.5, 1.5, 1.3, and 1.18 are shown. At the highest threshold, see Fig. 3(a), the well defined features due to the $\mathrm{H}$-bonded nearest neighbors (containing two molecules) are evident; the single feature below the nitrogen contains a single neighbor, while each of the two caps centered over the amine hydrogens combine to account for the second H-bonded nearest neighbor. Clearly, the methylamine appears to have only two H-bonded nearest neighbors (as determined geometrically).

As we begin to lower the isosurface threshold in Figs. 3(b) and 3(c), we see somewhat more distant, but still near neighbor, structures appearing above and in front of the amino group. These new features account for some of the additional neighbors found in the first peak of the N-N RDF. From an additional analysis in which we have added to Fig.

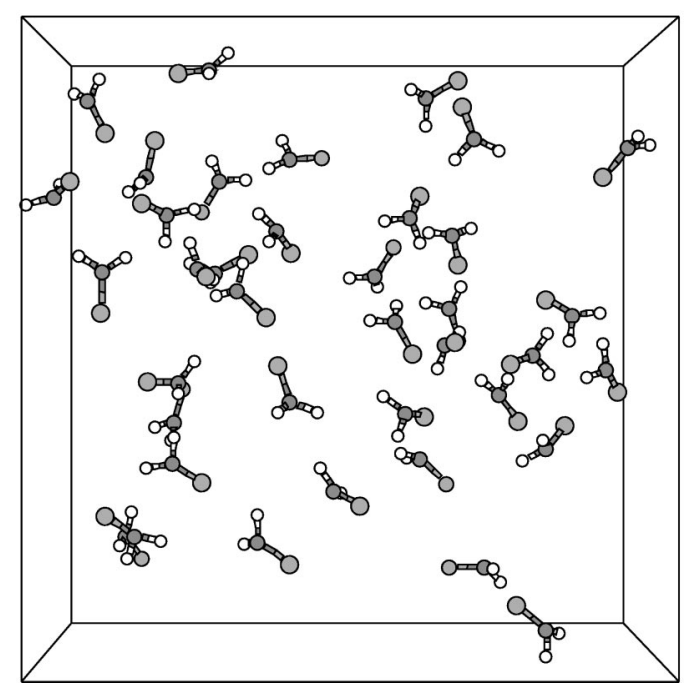

FIG. 4. Instantaneous configuration of methylamine molecules from a 30 wt $\%$ simulation. All water molecules are removed for clarity.

3(b) the neighboring hydrogen local density around the methylamine molecule, we find that the feature above the amino group is due to a $\mathrm{H}$-bond donor neighbor that is forming a longer (weaker) H-bond to the "back-side" of the nitrogen atom. Further, the hydrogen density associated with the nitrogen "nose" appearing in front of the amino group lies, in fact, at smaller separations indicating that this is not a dipolar or bifurcated H-bond arrangement, but rather is an opposed neighbor as one might have in a small (i.e., fourmember) ring-like structure. We remark that evidence for small (three- or four-member) ring-like arrangements can be seen from the detailed examination of an instantaneous molecular configuration of the liquid (see Fig. 4).

The extension of the principal H-bonding structures to larger separations (lighter shading) indicates that some molecules positioned over H-bonding sites either do not participate in or participate in weak (longer) H-bonds, such as back-side or bifurcated H-bonds, from these positions. The "wings" that develop at larger separations on the edge of the principal H-bond donor feature below the molecule are evidence of such bifurcated arrangements. We point out that this arrangement in liquid methylamine is reminiscent of the crystal structure $^{42}$ where each methylamine molecule participates in two bifurcated H-bonds. Note that the more distant feature below the amino group results from second neighbors. Thus we see that beyond some similarity in principal $\mathrm{H}$-bond features, the spatial structure in methanol ${ }^{33}$ (where beyond the $\mathrm{H}$-bonded first neighbors one finds clear evidence of second H-bonded and interchain neighbors) and methylamine are distinctly different. As a double H-bond donor but single acceptor the compromises that methylamine makes in the nearest-neighbor structure around the amino group are rather striking; on average each nitrogen maintains four near neighbors where only two of these are (strongly) H-bonded and the other two neighbors attempt much weaker H-bonds.

At the lowest isosurface threshold displayed in Fig. 3(d), we see nitrogen density resulting from methylamine neighbors around the methyl group of the central molecule. Of significance is the fact that even at this rather low threshold, 


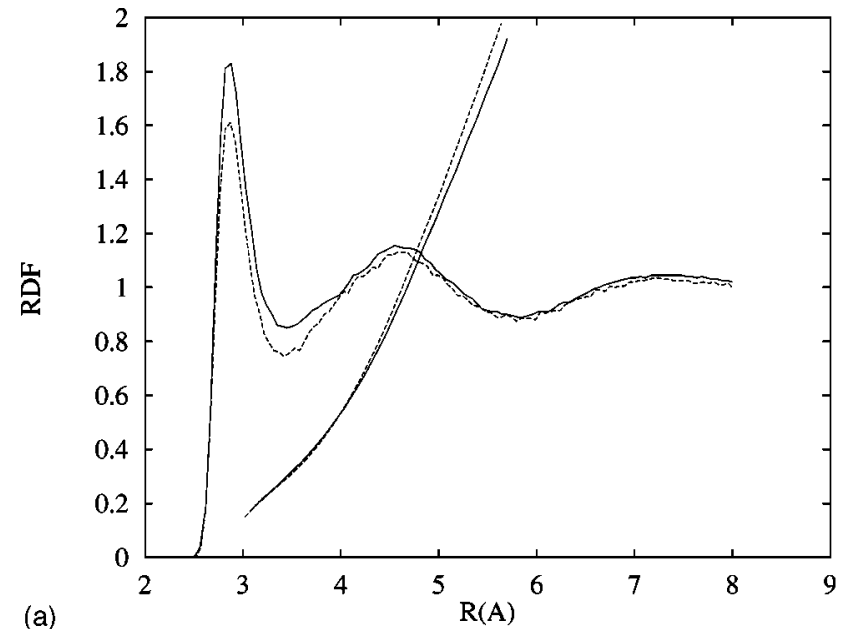

(a)

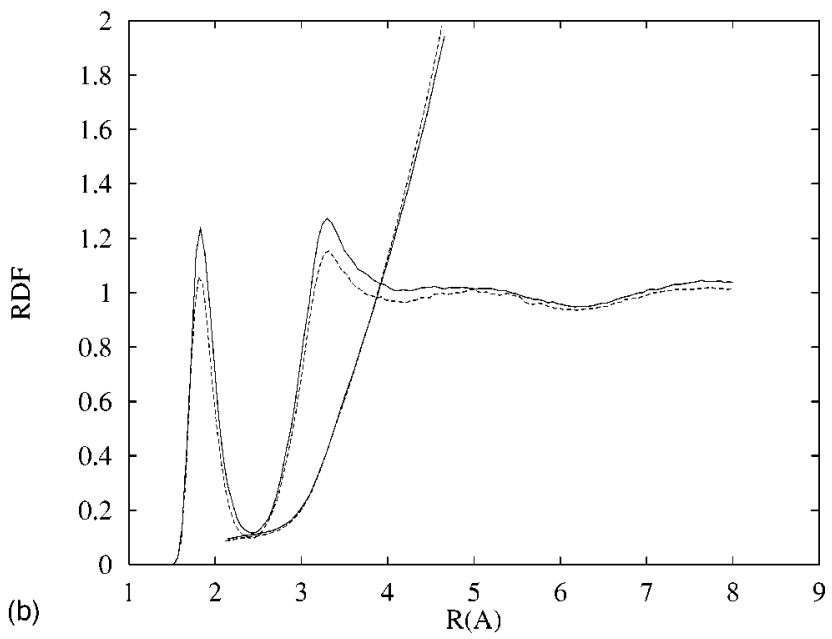

FIG. 5. Radial distribution functions between (a) nitrogen-oxygen, and (b) nitrogen-water hydrogen in aqueous methylamine solution. The solid line corresponds to a $10 \mathrm{wt} \%$ solution and the dotted line to a $30 \mathrm{wt} \%$ solution. Lines representing running coordination numbers around the nitrogen have also been included on a 1/10 scale.

no secondary coordination structures (due to second H-bonded neighbors) have appeared around the amino group. Taken together with other SDF results (e.g., the methyl-methyl SDF, not shown), we conclude that the structure in liquid methylamine can be characterized as being somewhat heterogeneous on a local scale, with a strong association of methyl (hydrophobic), separate from amino $(\mathrm{H}-$ bonding), groups. In particular, amino groups appear to tend to cluster together in small compact ring or ring-like arrangements. This is in contrast to methanol ${ }^{33}$ where open, nonlinear H-bonded chains are the dominant liquid structure.

\section{B. Structure in aqueous methylamine solution}

\section{Solution structure based on radial analysis}

In Fig. 5 the functions $g\left(r_{\mathrm{NO}}\right)$ and $g\left(r_{\mathrm{NH} w}\right)$ are displayed at the two concentrations investigated along with running values for the coordination numbers around the nitrogen. From Fig. 5(a) we see that both RDF curves have a distinct peak at $2.85 \AA$, corresponding to apparently strong $\mathrm{N}-\mathrm{H} \cdots \mathrm{O}$ and $\mathrm{O}-\mathrm{H} \cdots \mathrm{N}$ hydrogen bonds. The intensity is somewhat higher for the curve for the $10 \mathrm{wt} \%$ solution. The

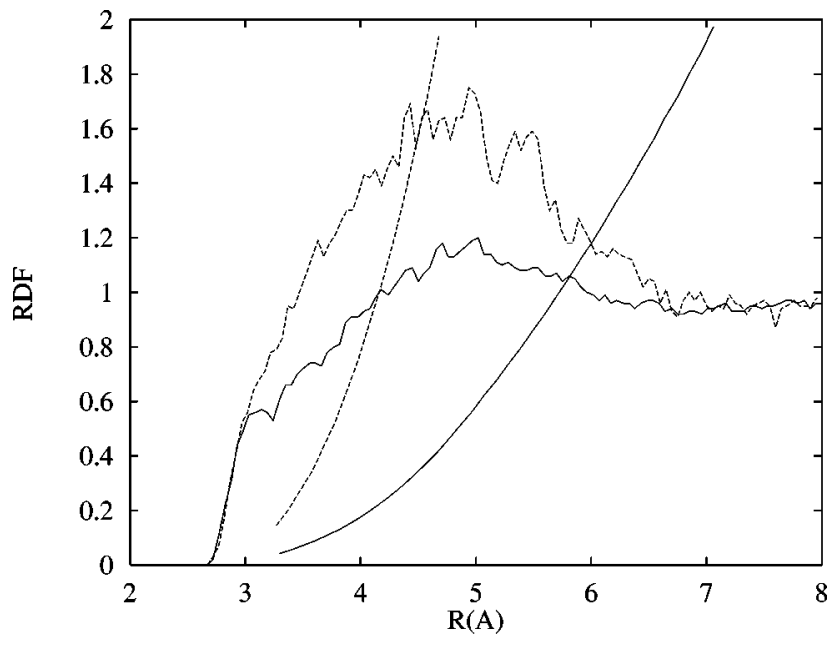

FIG. 6. Radial distribution functions between nitrogen-nitrogen in aqueous methylamine solution. The lines are defined as in Fig. 5.

curves representing $g\left(r_{\mathrm{NH} w}\right)$ in Fig. 5(b) confirm a strong hydrogen bond between a nitrogen and water hydrogen, with the maxima at $1.9 \AA$. The first minima reach a value of 0.10 indicating a fairly stable solute-solvent $\mathrm{H}$-bond when methylamine acts as the acceptor. With the downward shift of both the RDF results in Fig. 5, we find that the water hydrogen and the oxygen coordination numbers for the first hydration shell are relatively unchanged with the three-fold increase in concentration.

The solute-solute RDF $g\left(r_{\mathrm{NN}}\right)$ for both solutions investigated are given in Fig. 6. The methylamine shows apparently only a slight degree of association in aqueous solution, with very few H-bonded pairs. Both curves in Fig. 6 show a maximum at roughly $5 \AA$, but it is rather weak in the 10 wt \% solution. These RDF results appear to suggest that methylamine prefers to $\mathrm{H}$-bond to water and that otherwise there is little specific solute-solute correlations. Note that a similar conclusion drawn from the methanol oxygen-methanol oxygen RDF for dilute aqueous methanol solution proved to be incorrect when the full spatial structure was examined. ${ }^{5}$

In Fig. 7, we consider RDFs of the water oxygen. $g\left(r_{\mathrm{OO}}\right)$ is displayed in Fig. 7, while the RDF between the oxygen and the methylamine hydrogens is shown in Fig. 7(b). Running values for the coordination numbers around the oxygen are also included in Fig. 7(a). We can see from Fig. 7(a) that the water-water correlations appear slightly reduced in the $30 \mathrm{wt} \%$ solution, although both solutions show a slightly more pronounced first peak relative to pure SPC/E water. ${ }^{35}$ Given this RDF evidence, one might speculate that the hydrophobic methyl groups could, as suggested for aqueous methanol solutions, induce formation of a cagelike hydration shell. It can been seen from Fig. 7(b) that the $\mathrm{O} \cdot \mathrm{H}-\mathrm{N}$ hydrogen-bond is apparently strong. Yet, although the first peak also has a maximum at roughly $1.9 \AA$ suggesting a H-bond similar in strength to that when water acts as the H-bond donor to the nitrogen [see Fig. 5(b)], the $\mathrm{O} \cdot \mathrm{H}-\mathrm{N}$ peak is also broader with a first minimum of about 0.4 .

Examination of the coordination numbers provided by the RDFs in Figs. 5 and 7 also reveals some intriguing be- 

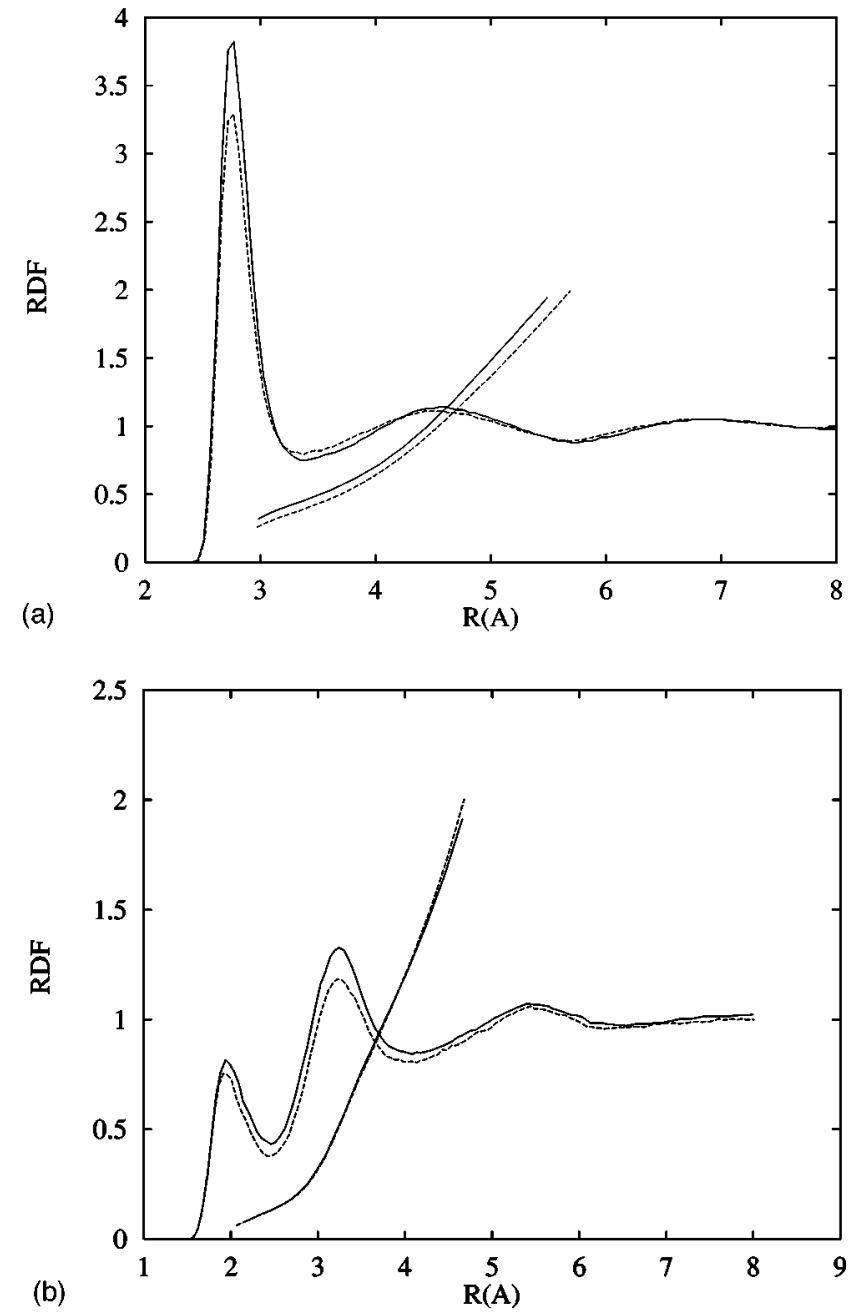

FIG. 7. Radial distribution functions between (a) oxygen-oxygen, and (b) oxygen-methylamine hydrogen in aqueous methylamine solution. The lines are defined as in Fig. 5, where the running coordination numbers are for neighboring atoms around the oxygen.

havior. The coordination numbers from the integration of the first peaks in the nitrogen-oxygen, nitrogen-water hydrogen, and oxygen-methylamine hydrogen, are essentially composition independent, virtually unaffected by a three-fold increase in the methylamine concentration. The local environment of each methylamine molecule thus appears to be effectively the same in the two solutions considered. Further, we find that [see Fig. 7(a)] the number of water molecules in the first-neighbor shell around the oxygen decreases somewhat, from 4.3 to 3.8 , on going to the more concentrated solution. From Fig. 5(a) there appears to be three water molecules (oxygen atoms) in the first hydration shell around the methylamine nitrogen; it may be tempting to assume then that all three of these water molecules are $\mathrm{H}$-bonded to the amino group. However, we see from Fig. 5(b) and Fig. 7(b) that only one of these water molecules accepts an H-bond and only one donates an $\mathrm{H}$-bond. Thus, the amino group appears to be making only two (strong) H-bonds with water. The status of the third near-neighbor water molecule is then left unclear. Dunn and Nagy ${ }^{21,22}$ have speculated that this third nearest-neighbor water may form a bridge between the two other H-bonding water molecules (see Fig. 9 of Ref. 22).

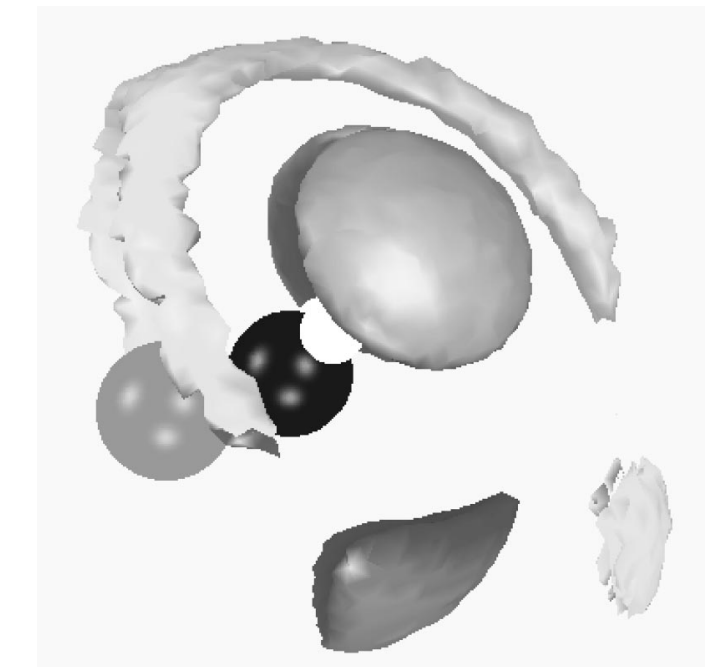

FIG. 8. Local water structure around methylamine as measured by the nitrogen-oxygen spatial distribution function. The isosurfaces shown are for a $30 \mathrm{wt} \%$ aqueous solution and an oxygen density threshold 1.75 times that of the bulk. The surfaces have been shaded so that those appearing lighter are further from the nitrogen.

Finally, we remark that our RDF results are completely in accord with previously published results. ${ }^{21,22}$

\section{Solution structure revealed by spatial distribution functions}

In our SDF analysis we have examined the site-specific local structure and its dependence on concentration. In general, we observe that the local structure was quite similar for the two solutions we have examined. Hence, the analysis below is valid for both compositions studied, unless explicitly noted otherwise.

Let us first focus on the local structure around the methylamine solute. In Fig. 8 the spatial distribution function of oxygen atoms around the methylamine is shown as threedimensional isosurfaces. For the particular isosurface density threshold displayed, 1.75 times the bulk value, we can see several key features. Clearly evident are the basic H-bonded features, as caps over each of the two hydrogens and as a slightly elongated (in and out of the page for the perspective shown in Fig. 8) triangular feature immediately below the amino group. These features resemble the corresponding ones found in pure methylamine (see Fig. 3), indicating some similarity in the basic H-bonding structure. The remaining (secondary) structure evident in Fig. 8 is due to second H-bond neighbors. The ridge extending between the two amino hydrogen caps is particularly interesting in that it represents a bridging second-neighbor water (i.e., it is H-bonded to both neighbors occupying H-bond accepting sites). However, it is important to note that this bridging feature only comes to within about $3.3 \AA$ of the nitrogen, and hence cannot effectively be part of the extra water coordination evident in the first peak of $g\left(r_{\mathrm{NO}}\right)$ [see Fig. 5(a)]. The origins of this 
extra coordination are manifest in Fig. 8 by the slightly lighter shading of the outer surfaces of the two caps over the amine hydrogens, relative to that of the first-neighbor $\mathrm{H}$-bond donor feature. We find that the latter extends out to distances of only about $3.2 \AA$, while the former structures include neighbors as distant as $3.8 \AA$. Moreover, integration over the H-bond donor feature reveals that it contains almost exactly one neighbor, while the two caps (when adjusted for the small $\mathrm{N}$ density present) together give a value approaching two (an exact value being difficult to determine due to overlaps with nearby features). Thus, the nitrogen is accepting a single strong $\mathrm{H}$-bond from water. In return, to maintain $\mathrm{H}$-bond balance, water chooses to accept on average only a single (strong) H-bond from the amino group; at any instant in time this H-bonded water occupies one of the caps in the local oxygen density and will be found at smaller separations $(2.8 \AA)$ appropriate of a H-bonded neighbor. However, the second cap is not left vacant, as this would leave a large hole in the local structure, but rather is occupied by a slightly more distant water molecule that is H-bonded to the local hydration structure (to a bridging water or possibly even the molecule in the other cap), yet does not (strongly) H-bond to the amino group. Another way perhaps to view this arrangement is to say that this water forms a weak bifurcated H-bond to the amino group which it shares with another (water) molecule. This structural picture then explains the RDF data discussed above; it is also supported by both the water-hydrogen (not shown) and the complimentary oxygen-nitrogen (Fig. 10, discussed below) SDF.

As was noted above in our RDF discussion, there is a relatively low probability of finding a methylamine $\mathrm{H}$-bonded to another methylamine in our aqueous solutions, with $g\left(r_{\mathrm{NN}}\right)$ showing only a very flat broad peak centered at about $5 \AA$ (see Fig. 6). Visualization of the nitrogennitrogen SDF (not shown) similarly reveals no particularly sharp or distinct structures. As expected, there are only minor $\mathrm{H}$-bond features in the nitrogen density immediately around the amino group and a large cap around the methyl group, due to hydrophobically associated methylamines (i.e., the local nitrogen density appears more distant than its associated methyl density). We remark that this behavior is in sharp contrast to that observed for dilute methanol solution where very distinct long-range spatial correlations were observed. $^{5}$

In Fig. 9 we consider the "hydrophobic" aspect of the structure around methylamine by examining the local methyl density. The helmet-like bowl that almost completely surrounds the methyl group at even the relatively high isosurface threshold (2.6) shown in Fig. 9 provides clear evidence of a strong hydrophobic association in the methylamine structure. This bowl, which opens toward the amino end of the molecule, contains methyl groups that are essentially in contact with the methyl group of the central molecule. However, a more detailed examination of this helmet-shaped feature shows that it contains no particularly distinct peaks, indicating that these hydrophobic methyl-to-methyl associations are not otherwise strongly directionally specific.

The local nitrogen density around a water molecule in aqueous methylamine solution is given in Fig. 10, where we

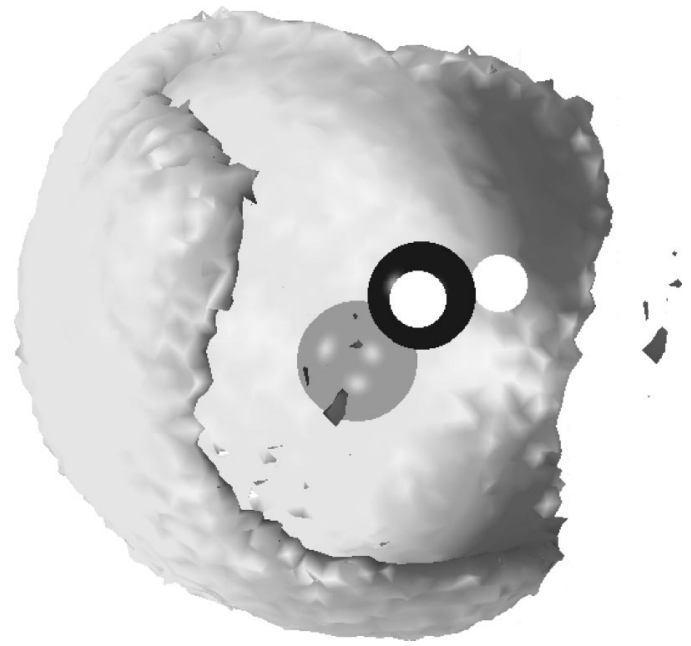

FIG. 9. The spatial distribution function of the local methyl density around methylamine. The isosurfaces shown are for a $30 \mathrm{wt} \%$ solution and an methyl density threshold 2.6 times that of the bulk.

have displayed the oxygen-nitrogen SDF at two isosurface thresholds. At the higher density threshold of 2, we can see four distinct H-bonded features in Fig. 10(a), two symmetrical caps over the hydrogens and two rather distorted (triangular) caps below the "lone-pair" sites. We remark that even at much lower density thresholds the gap between the two H-bond donor structures below that water molecule still remains, indicating that water does not adopt a dipolar ori-

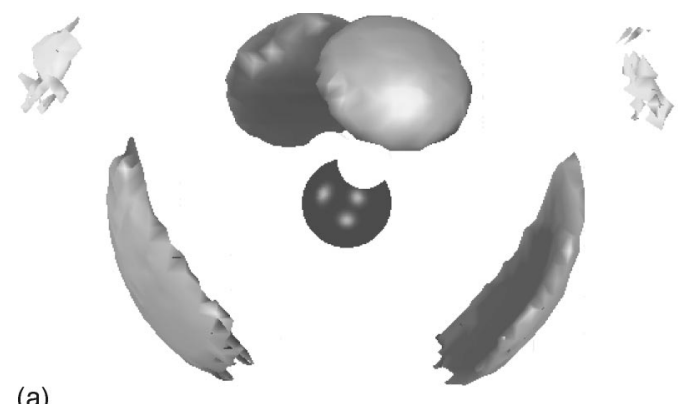

(a)

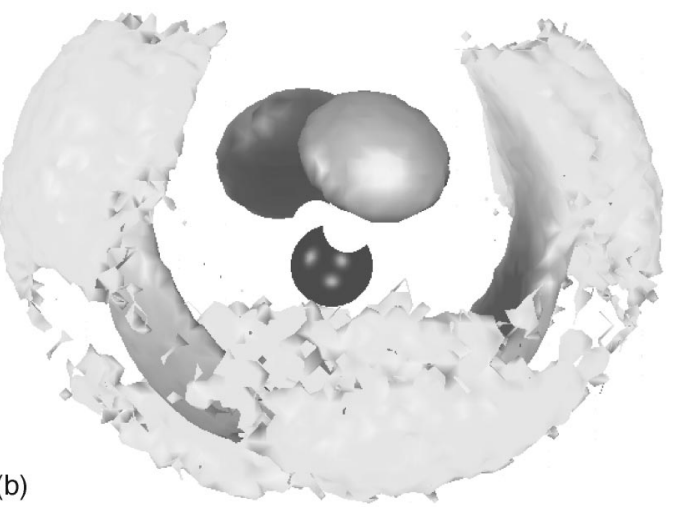

FIG. 10. Spatial distribution functions of the local nitrogen density around water in a $10 \mathrm{wt} \%$ aqueous solution. The isosurfaces threshold densities are 2 and 1.55 times the bulk value in (a) and (b), respectively. The surfaces have been shaded so that those appearing lighter are further from the water oxygen. 
entation with respect to the nitrogen, but rather strongly prefers to use both of its H-bond acceptor (lone-pair) sites. This behavior can be understood by again considering the balance of the numbers of $\mathrm{H}$-bond donor and acceptor sites in these methylamine solutions. With an excess of donor sites, it would seem that water, particularly when it $\mathrm{H}$-bonds to the nitrogen, will use both its acceptor sites.

Examination of Fig. 10(a) also reveals two other features, perpendicular to the plane of the molecule and slightly above its equator, lighter in shading indicating that they are more distant. This is the first suggestion of much larger structures that appear at lower thresholds, as in Fig. 10(b), corresponding to the nitrogen of a neighboring methylamine with its methyl group pointing at the water (or equivalently, the central water molecule lying near and perpendicular to the surface of the methyl group). Similar features were noted in aqueous methanol solutions. ${ }^{5}$ The light gray structures appearing near the plane of the molecule, slightly below its equator and less well defined, correspond to the analogous symmetry related arrangements of a water next to a methyl group. It is also important to point out that the two rather bulky out-of-plane more lightly shaped features that can be seen in Fig. 10(b) extend over a very large range of separations, $3.1 \AA$ to $4.9 \AA$; they in fact contain nitrogen densities associated with three different kinds of methylamine neighbors. At the smallest separations and connected continuously to the $\mathrm{H}$-bond donor feature, is nitrogen density due to a nearest-neighbor methylamine that does not (strongly) $\mathrm{H}$-bond to the central water. This unique association of a water molecule with an amino hydrogen, which one could refer to as a "weak H-bond," is the same as we discussed in Fig. 8 above. At the most distant separations $(>4.2 \AA)$ is nitrogen density due to the water molecules lying next to the methyl surface, as first evident in Fig. 10(a). The nitrogen density at intermediate separations (roughly $4 \AA$ ) is due to the central water molecule playing the role of a bridging second neighbor to the amino group, a feature that was also clear from Fig. 8. Finally, it is interesting to point out that while the SDFs in Figs. 8 and 10 appear quite dissimilar, reflecting the unique local arrangements around the nitrogen and oxygen atoms, respectively, they will nonetheless angleaverage to the same radial distribution function [see Fig. $5(\mathrm{a})$ ]; here the loss of information on angle-averaging is particularly striking.

We examine the local structure of water around the hydrophobic methyl group in Fig. 11. In Fig. 11(a) the oxygenmethyl SDF is displayed at a relatively high isosurface threshold of 2.7, while in Fig. 11(b) the result is overlayed with the oxygen-nitrogen SDF from Fig. 10. It can be clearly seen that the water molecules adopt specific orientations when next to a methyl group (i.e., these four methyl features appear in near equatorial positions, two slightly above and two slightly below the equator, and at smaller separations than their associated nitrogen densities apparent in Fig. 10). We can also confirm from Fig. 11 that the ring of methyl density above the hydrogen is due to a H-bonded methylamine neighbor. The specific relative orientations of the two molecules and the molecular geometry of the meth-

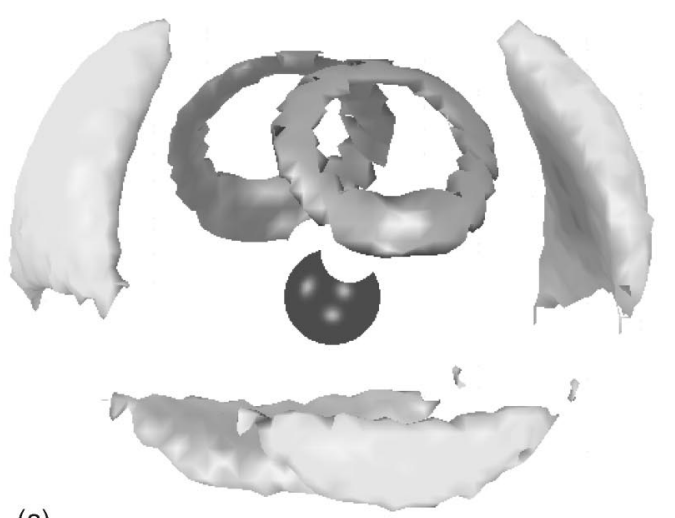

(a)

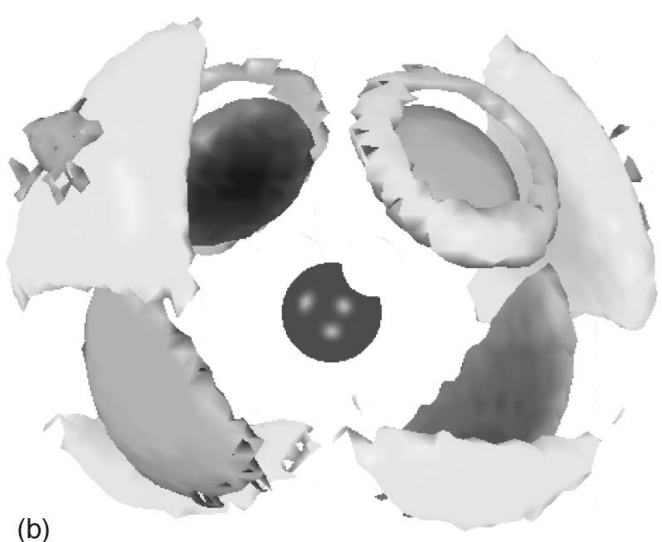

FIG. 11. Local methylamine density around water in a $10 \mathrm{wt} \%$ solution. (a) Isosurfaces for the local methyl density for a threshold of 2.7 times the bulk value are shown. (b) Isosurfaces for the local nitrogen density around oxygen for a threshold of 2 times the bulk have been overlayed with the methyl isosurfaces from (a), where the features for the nitrogen have been shaded to appear darker.

ylamine give rise to the somewhat unusual ring-shaped feature.

Finally, in Fig. 12 we show the oxygen-oxygen SDF for the $30 \mathrm{wt} \%$ solution of methylamine. We see some expected

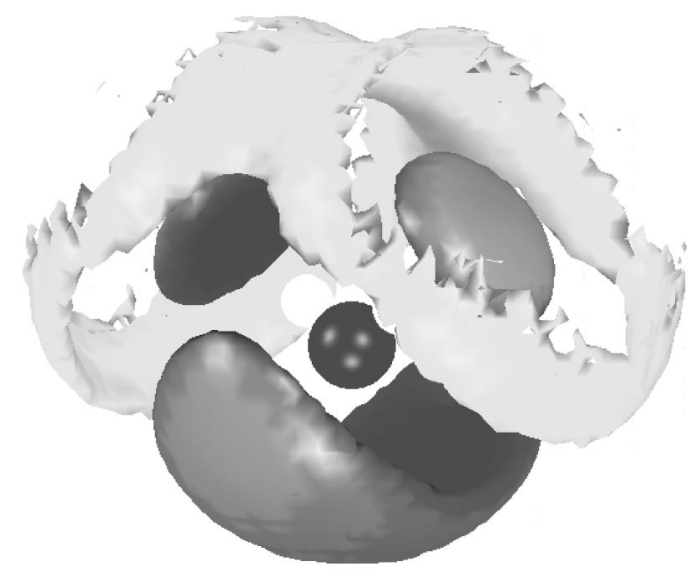

FIG. 12. Local water structure around a water molecule in the $30 \mathrm{wt} \%$ solution as measured by the oxygen-oxygen SDF. The isosurfaces shown are for an oxygen density threshold 1.5 times that of the bulk. The features appearing lightly shaded are due to more distant second neighbors. 

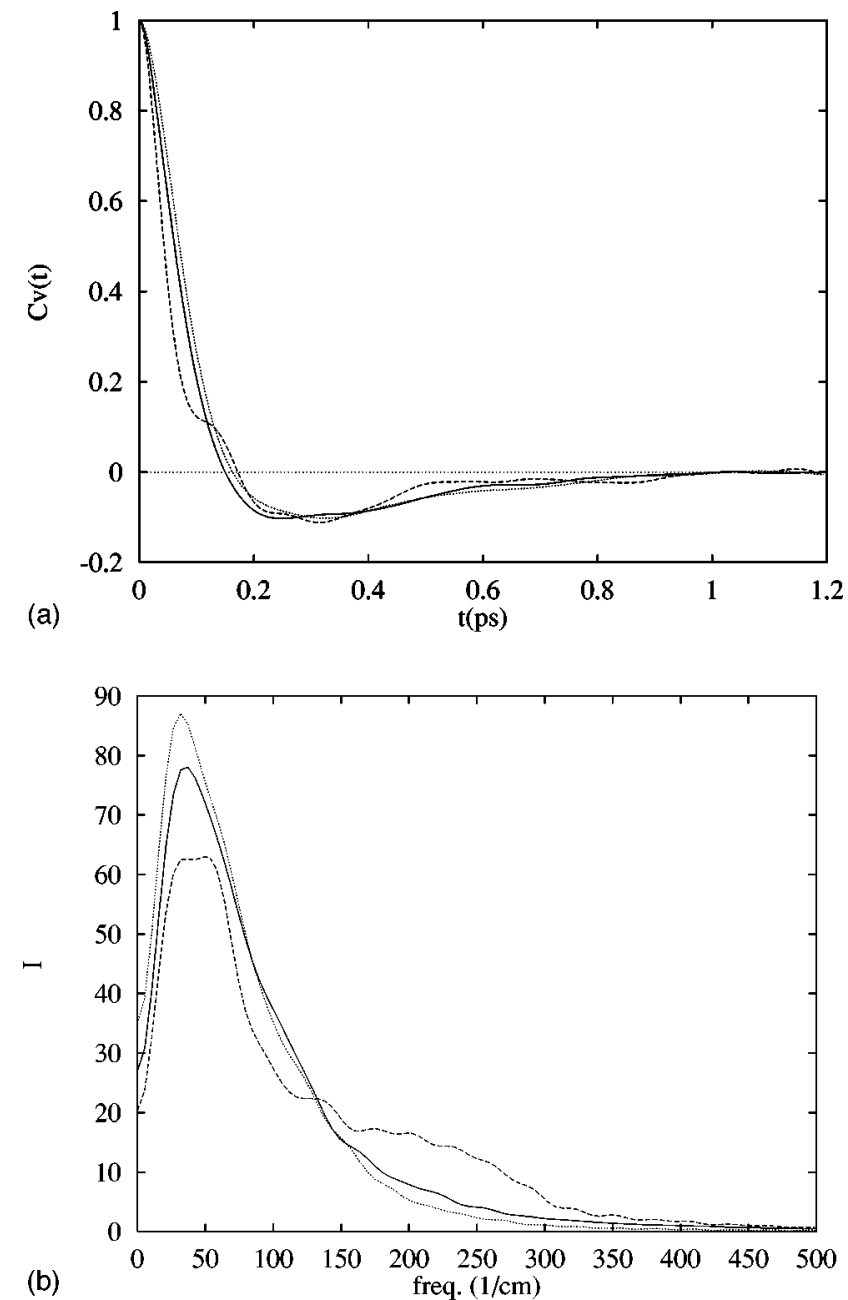

FIG. 13. (a) Linear velocity autocorrelation function for methylamine, decomposed into its three molecular frame components, for the $30 \mathrm{wt} \%$ solution. (b) Power spectra of the components of the linear velocity autocorrelation function for methylamine. The solid, dotted and dashed lines are the $X, Y$, and $Z$ components, respectively.

features in the local water structure, the darker nearestneighbor and the lighter second $\mathrm{H}$-bonded neighbor features. Missing from the water structure is the interstitial density feature characteristic of the pure liquid. ${ }^{31}$ The fact that the secondary structure is already well resolved at a density threshold of 1.5 is an indication of an enhancement of the local water-water correlations in the solution relative to that of the pure liquid. As we would expect, the enhancement is less pronounced in the $10 \mathrm{wt} \%$ solution.

\section{Dynamical aspects of hydration}

\section{Translational motion}

Translational velocity autocorrelation functions (VACF),

$$
\hat{C}_{v}(t)=\frac{\langle\overrightarrow{v(t)} \cdot \overrightarrow{v(0)}\rangle}{\langle\overline{v(\overrightarrow{0})} \cdot \overrightarrow{v(\overrightarrow{0})}\rangle},
$$

for both water and methylamine have been calculated in our simulation runs. In Fig. 13 we report results only for methylamine in the $30 \mathrm{wt} \%$ solution. In Table III, we give the calculated correlation times from the direct integration of the
TABLE III. Dynamical data calculated from the linear velocity autocorrelation functions. Errors in this data are estimated to be less than 5\%.

\begin{tabular}{lccc}
\hline \hline \multicolumn{1}{c}{ Quantity } & $\begin{array}{c}10 \mathrm{wt} \% \text { of } \\
\mathrm{MeNH}_{2}\end{array}$ & $\begin{array}{c}30 \mathrm{wt} \% \text { of } \\
\mathrm{MeNH}_{2}\end{array}$ & $\begin{array}{c}\text { Pure liquid } \\
\mathrm{MeNH}_{2}\end{array}$ \\
\hline $\mathrm{H}_{2} \mathrm{O}$ & & & \\
$\tau_{v}-\mathrm{X}(\mathrm{ps})$ & 0.015 & 0.013 & $\ldots$ \\
$\tau_{v}-\mathrm{Y}(\mathrm{ps})$ & 0.023 & 0.020 & $\cdots$ \\
$\tau_{v}-\mathrm{Z}(\mathrm{ps})$ & 0.013 & 0.011 & $\cdots$ \\
$\mathrm{D}_{v}\left(10^{9} \mathrm{~m}^{2} / \mathrm{s}\right)$ & 2.30 & 1.96 & $\cdots$ \\
$\mathrm{CH}_{3} \mathrm{NH}_{2}$ & & & \\
$\tau_{v}-\mathrm{X}(\mathrm{ps})$ & 0.030 & 0.027 & 0.065 \\
$\tau_{v}-\mathrm{Y}(\mathrm{ps})$ & 0.020 & 0.018 & 0.059 \\
$\tau_{v}-\mathrm{Z}(\mathrm{ps})$ & 0.042 & 0.035 & 0.069 \\
$\mathrm{D}_{v}\left(10^{9} \mathrm{~m}^{2} / \mathrm{s}\right)$ & 2.36 & 2.16 & $4.31(\text { Exp. } 5.10)^{\mathrm{a}}$ \\
$\tau_{\omega}-\mathrm{X}(\mathrm{ps})$ & $\cdots$ & 0.0054 & $\cdots$ \\
$\tau_{\omega}-\mathrm{Y}(\mathrm{ps})$ & $\cdots$ & 0.0230 & $\cdots$ \\
$\tau_{\omega}-\mathrm{Z}(\mathrm{ps})$ & $\cdots$ & 0.0010 & $\cdots$ \\
\hline \hline
\end{tabular}

${ }^{\mathrm{a}}$ Chen et al. (Ref. 44).

VACFs, resolved into the three directional components of the molecular coordinate system. The self-diffusion coefficients for both solution components were also computed from the autocorrelation functions. From Table III we see that, for example, water molecules are most mobile in the $Y$-direction, or perpendicular to the molecular plane, as is the case in the pure liquid. ${ }^{31}$ The translational diffusive motion of water molecules is somewhat slower in the more concentrated solution, which is consistent with the slight increase in water structure noted for these solutions in our discussion above.

The self-diffusion coefficients for methylamine in aqueous solution are markedly smaller than in the pure liquid, becoming rather similar in magnitude to those for water. In the pure liquid there is only a slight assymetry in the local diffusive motion, while in aqueous solution methylamine is considerably more mobile along its principal $(Z)$ axis, and its motion is most hindered in $Y$-direction (see Fig. 1). The $Y$-component of the VACF [see Fig. 13(a)] is also most different from the pure liquid result, where the small amplitude oscillations with a $0.1 \mathrm{ps}$ period are not present. We also note that all three components of the pure liquid VACF lack the strong rebound (or cage effect) ${ }^{43}$ feature present in the solution curves at roughly $0.3 \mathrm{ps}$. Clearly the hydration of this molecule is strongly altering its translational dynamics, and particularly the $Y$-component seems to be the most influenced by the changes in local structure.

In Fig. 13(b), we display the power spectra of the velocity autocorrelation functions obtained by direct Fourier transform. The correlation times (see Table III) can then be read as the zero-frequency values (in femtoseconds). All three power spectra have a dominant mode around $35 \mathrm{~cm}^{-1}$. The $Y$-component does, however, show a weak splitting and a shoulder at about $200 \mathrm{~cm}^{-1}$, again not present in the pure liquid, suggesting some coupling to the solvent modes.

\section{Angular motion}

The angular velocity time correlation functions (AVACF), 

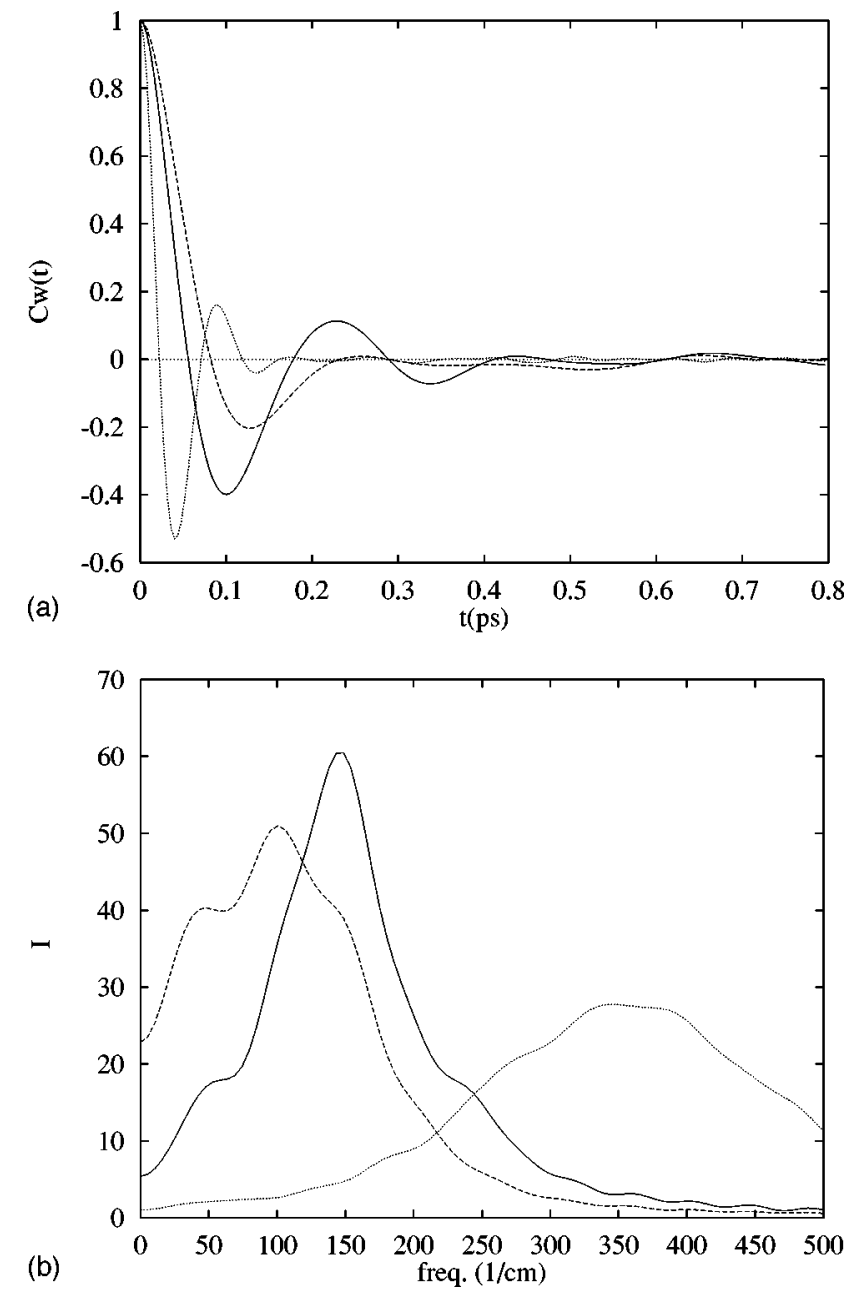

FIG. 14. (a) Angular velocity autocorrelation function for methylamine, decomposed into its three molecular frame components, for the $30 \mathrm{wt} \%$ solution. (b) Power spectra of the components of the angular velocity autocorrelation function for methylamine. The solid, dashed, and dotted lines are the $X, Y$, and $Z$ components, respectively.

$$
\hat{C}_{\omega}(t)=\frac{\langle\overrightarrow{\omega(t)} \cdot \overrightarrow{\omega(0)}\rangle}{\langle\overline{\omega(\overline{0})} \cdot \overline{\omega(\overline{0})}\rangle},
$$

were calculated for the $30 \mathrm{wt} \%$ solution. The procedure was the same as that used to obtain the VACF except that the angular velocities about the principal axes of the molecules were used in place of the components of the linear velocity. Again we find a high degree of assymetry, now in the angular motion of methylamine molecule. In Fig. 14 we show these angular time correlation functions for methylamine in this solution, where the components can be seen to be quite different. However, when one takes into account the assymetry in the moment of inertia tensor (roughly: $I_{x x}=18.7$, $I_{y y}=20.0$, and $I_{z z}=1.5$ ) and assumes a simply square-root inertial dependence, it is apparent that the $X$ - and $Z$-components are more similar than they immediately appear. The $Y$-component continues to stand out, now because of its large correlation time [equal to the zero frequency value in Fig. 14(b)] and more damped functional behavior. Yet it is interesting to point out that it is the $X$-component of the angular velocity time correlation function that differs the most from the pure liquid result.
As expected the rotational motion of the methylamine molecule has considerably higher frequencies compared to the translational motion. From the power spectra, shown in Fig. 14(b), we observe the strongest modes at 150 and 100 $\mathrm{cm}^{-1}$ for rotations around $X$ - and $Y$-axes, respectively, while for motion about the $Z$-axis, the principal mode appears at a much higher frequency of $350 \mathrm{~cm}^{-1}$. Both the $X$ - and $Y$-components exhibit shoulders at $50 \mathrm{~cm}^{-1}$, again suggestive of a coupling to solvent (water) modes.

\section{CONCLUSIONS}

In this paper we have reported computer simulation results exploring in detail the local structure in liquid methylamine and in aqueous methylamine solutions. Molecular dynamics simulations were carried out for model systems of the pure liquid and of two aqueous solutions representing mixtures containing 10 and $30 \mathrm{wt} \%$ methylamine. Radial distributions functions (RDF) were employed to aid in our structural analysis, however spatial distribution functions (SDF), correlation functions that span the full three-dimension space surrounding a molecule, were used to provide more detailed information about the local environments in these liquid systems. As in previous work, it was found that the SDFs provide a much more complete and unambiguous picture of the local liquid structure. Dynamical properties were also examined to provide further structural insights.

In pure liquid methylamine, integration of the first peak of the nitrogen-nitrogen RDF revealed roughly four neighboring nitrogen atoms. Yet, from the nitrogen-hydrogen RDF we find that each methylamine molecule makes only two (strong) H-bonds. Using SDFs, we have been able to show that the remaining nitrogen coordination is due to more distant amino groups that are either weak or non-H-bonding first neighbors, or closely lying second neighbors. Beyond these near neighbors, however, there is little secondary structure evident around the amino group. We do find a strong association of methyl groups in the liquid methylamine. Despite a similarity between the RDFs, as was noted by previous authors, ${ }^{36}$ our SDFs clearly show that the local structure in methylamine is quite unlike the chaining motif that appears to dominate the structures found in liquid methanol. Rather the molecules appear to prefer arrangements with a few amino groups clustered together in small compact ring or ring-like structures.

We did not observe a strong composition dependence in the local structure for the two aqueous methylamine solutions examined. We again find that the rather limited information provided by RDFs is not sufficient to resolve fully the complex local structure in these solutions. Two primary $\mathrm{H}$-bonded water molecules, one as an accepting neighbor and one as a donating neighbor, were found. Even more interestingly, we observe that the second $\mathrm{H}$-bond accepting site of the amino group is not vacant, but rather is occupied on average by a slightly more distant nearest-neighbor water that forms only a weak H-bond to the amino hydrogen, preferring instead to H-bond to the surrounding hydration network. To our knowledge, this quite unique hydration structure has not been detected before. Additional coordination due to bridging water molecules was also evident, but we 
find that previous analysis ${ }^{21,22}$ that attempted to include this water in the first peak of $g\left(r_{\mathrm{OO}}\right)$ is incorrect. A strong hydrophobic association of methyl groups was observed in these aqueous solutions but no long range correlations of methylamine molecules are apparent, as is the case found in dilute methanol solution. ${ }^{5}$ We did find that water molecules around the methyl group tend to lay flat against its hydrophobic surface. Our SDF analysis does reveal some increased ordering in the water-water structure, although not to the extent found dilute methanol solution. ${ }^{5}$

We have shown that within even relatively simple aqueous nonelectrolyte solutions the molecular arrangements can be both complex and unexpected; we have again demonstrated the ability of SDFs to provide detailed insights into the local structure within such systems. As in previous work with methanol-water mixtures, ${ }^{5}$ we do find that H-bond balance appears to play a key role in determining the preferred local structures around H-bonding molecules. It may now be particularly interesting to observe the local structures preferred in aqueous systems with both alcohol and amino groups present, where we might suspect a high degree of cooperativity.

\section{ACKNOWLEDGMENTS}

This work has been supported by the Swedish Natural Science Research Council (NFR) and by the Natural Science and Engineering Research Council of Canada (NSERC).

${ }^{1}$ W. Saenger, Principles in Nucleic Acid Structure (Springer-Verlag, Heidelberg, 1984).

${ }^{2}$ G. A. Jeffrey and W. Saenger, Hydrogen Bonding in Biological Structures (Springer-Verlag, Heidelberg, 1991).

${ }^{3}$ P. G. Kusalik, A. Laaksonen, and I. M. Svishchev, Molecular Dynamics: From Classical to Quantum Methods, Theoretical and Computational Chemistry Series (Elsevier, Amsterdam, 1999), Vol. 7, Chap. 3, pp. 6198.

${ }^{4}$ A. Laaksonen, P. Stilbs, and R. E. Wasylishen, J. Chem. Phys. 108, 455 (1998).

${ }^{5}$ A. Laaksonen, P. G. Kusalik, and I. M. Svishchev, J. Phys. Chem. 101, 5910 (1997).

${ }^{6}$ H. Kovacs and A. Laaksonen, J. Am. Chem. Soc. 113, 5596 (1991).

${ }^{7}$ D. L. Bergman and A. Laaksonen, Mol. Simul. 20, 245 (1998).

${ }^{8}$ D. L. Bergman, L. Laaksonen, and A. Laaksonen, J. Mol. Graph. Modell. 15, 301 (1998).
${ }^{9}$ D. L. Bergman and A. Laaksonen, Phys. Rev. E 58, 4706 (1998).

${ }^{10}$ A. Laaksonen and P. Stilbs, Mol. Phys. 74, 747 (1991).

${ }^{11}$ A. Laaksonen and H. Kovacs, Can. J. Chem. 72, 2278 (1994).

${ }^{12}$ A. Lyubartsev and A. Laaksonen, J. Phys. Chem. 100, 16410 (1996).

${ }^{13}$ M. Odelius and A. Laaksonen, Molecular Dynamics: From Classical to Quantum Methods, Theoretical and Computational Chemistry (Elsevier, Amsterdam, 1999), Vol. 7, Chap. 8, pp. 281-324.

${ }^{14}$ J. A. Nilsson, L. A. Eriksson, and A. Laaksonen, J. Chem. Phys. 109, 2403 (1998)

${ }^{15}$ A. Vishnyakov, G. Widmalm, J. Kowalewski, and A. Laaksonen, J. Am. Chem. Soc. 121, 5403 (1999).

${ }^{16}$ K. Kulinska and A. Laaksonen, J. Biomol. Struct. Dyn. 11, 1307 (1994).

${ }^{17}$ A. Lyubartsev and A. Laaksonen, J. Biomol. Struct. Dyn. 16, 175 (1998).

${ }^{18}$ Industrial Solvents Handbook, edited by E. Flick (Noyes Data Corp., New Jersey, 1990).

${ }^{19} \mathrm{C}$. Reichardt, Solvents and Solvent Effects in Organic Chemistry $(\mathrm{VCH}$, Weinheim, 1988).

${ }^{20}$ M. S. B. Munson, J. Am. Chem. Soc. 87, 2332 (1965).

${ }^{21}$ W. Dunn and P. Nagy, J. Phys. Chem. 94, 2099 (1990).

${ }^{22}$ W. Dunn, P. Nagy, and E. Collantes, J. Am. Chem. Soc. 113, 7898 (1991).

${ }^{23}$ P. Nagy, Acta Chim. Hung. 129, 429 (1992).

${ }^{24}$ M. Kawata, S. Ten-no, S. Kato, and F. Hirata, Chem. Phys. 203, 53 (1996)

${ }^{25}$ Y. Ding, D. N. Bernardo, K. Krogh-Jespersen, and R. Levy, J. Phys. Chem. 99, 11575 (1995).

${ }^{26}$ P. Y. Morgantini and P. A. Kollman, J. Am. Chem. Soc. 117, 6057 (1995).

${ }^{27}$ E. C. Meng, J. W. Caldwell, and P. A. Kollman, J. Phys. Chem. 100, 2367 (1996).

${ }^{28}$ M. M. Kubo, E. Gallicchio, and R. M. Levy, J. Phys. Chem. B 101, 10527 (1997).

${ }^{29}$ T. I. Spector and P. A. Kollman, J. Phys. Chem. B 102, 4004 (1998).

${ }^{30}$ C. R. Rizzo and W. L. Jorgensen, J. Am. Chem. Soc. 121, 4827 (1999).

${ }^{31}$ I. M. Svishchev and P. G. Kusalik, J. Chem. Phys. 99, 3049 (1993).

${ }^{32}$ I. M. Svishchev and P. G. Kusalik, Science 265, 1219 (1994).

${ }^{33}$ I. M. Svishchev and P. G. Kusalik, J. Chem. Phys. 100, 5165 (1994).

${ }^{34}$ D. L. Bergman, Chem. Phys. 253, 267 (2000).

${ }^{35}$ H. J. C. Berendsen, J. R. Grigera, and T. P. Straatsma, J. Phys. Chem. 91, 6269 (1987)

${ }^{36}$ R. Impey, M. Sprik, and M. Klein, J. Am. Chem. Soc. 109, 5900 (1987).

${ }^{37}$ W. L. Jorgensen, J. Phys. Chem. 90, 1276 (1986).

${ }^{38}$ W. L. Jorgensen and J. M. Briggs, Mol. Phys. 63, 547 (1988).

${ }^{39}$ M. P. Allen and D. J. Tildesley, Computer Simulation of Liquids (Oxford Academic, Oxford, 1986).

${ }^{40}$ A. Laaksonen, Comput. Phys. Commun. 42, 271 (1986).

${ }^{41}$ A. K. Soper, Discuss. Faraday Soc. 103, 41 (1996).

${ }^{42}$ M. Atoji and W. N. Lipscomb, Acta Crystallogr. 6, 770 (1953).

${ }^{43}$ R. Lynden-Bell, Molecular Liquids: Dynamics and Interactions, NATO ASI Series (Reidel, Dordrecht, 1984), Vol. C135, pp. 501-518.

${ }^{44}$ L. Chen, T. Gross, and H.-D. Lüdemann, Phys. Chem. Chem. Phys. 1, 3503 (1999). 